\title{
Adaptive locomotor network activation during randomized walking speeds using functional near-infrared spectroscopy
}

\author{
Ha Yeon Kim ${ }^{\mathrm{a}, \mathrm{b}}$, Eun Joo Kim ${ }^{\mathrm{c}, 1, *}$ and Joshua (Sung) H. You ${ }^{\mathrm{b}, 1, *}$ \\ ${ }^{a}$ Translational Research Center for Rehabilitation Robots, National Rehabilitation Center, Seoul, Korea \\ ${ }^{\mathrm{b}}$ Sports $\cdot$ Movement $\cdot$ Artificial Robotics $\cdot$ Technology (SMART) Institute, Department of Physical \\ Therapy Program, Yonsei University, Wonju, Kangwon-do, Korea \\ ${ }^{\mathrm{c}}$ Department of Rehabilitation Medicine, National Rehabilitation Center, Seoul, Korea
}

\begin{abstract}
.
BACKGROUND: An improved understanding of the mechanisms underlying locomotor networks has the potential to benefit the neurorehabilitation of patients with neurological locomotor deficits. However, the specific locomotor networks that mediate adaptive locomotor performance and changes in gait speed remain unknown.

OBJECTIVE: The aim of the present study was to examine patterns of cortical activation associated with the walking speeds of $1.5,2.0,2.5$, and $3.0 \mathrm{~km} / \mathrm{h}$ on a treadmill.

METHODS: Functional near-infrared spectroscopy (fNIRS) was performed on a 30-year-old right-handed healthy female subject, and cerebral hemodynamic changes were observed in cortical locomotor network areas including the primary sensorimotor cortex (SMC), premotor cortex (PMC), supplementary motor area (SMA), prefrontal cortex (PFC), and sensory association cortex (SAC). The software package NIRS-statistical parametric mapping (NIRS-SPM) was utilized to analyze fNIRS data in the MATLAB environment. SPM t-statistic maps were computed at an uncorrected threshold of $p<0.00001$.

RESULTS: At faster walking speeds, oxygenated hemoglobin $(\mathrm{OxyHb})$ was concentrated in the PFC and indicated globalized locomotor network activation of the SMC, PMC, SMA, and PMC; additionally, the site with the highest cortical activation ratio shifted from the SMC to the SMA.

CONCLUSIONS: Global locomotor network recruitment, in particular PFC activation indicated by OxyHb in our study, may indicate a response to increased cognitive-locomotor demand due to simultaneous postural maintenance and leg movement coordination
\end{abstract}

Keywords: Functional near-infrared spectroscopy, gait speed, neuroimaging signal processing

\section{Introduction}

Walking velocity is an important functional parameter in victims of hemiparetic stroke due to lesioning of the contralateral sensorimotor cortex. Hemiplegic gait in patients of hemiparetic stroke is characterized by decreased gait speed. An enhanced understanding of the mechanisms underlying the locomotor

\footnotetext{
${ }^{1}$ Both Joshua (Sung) Hyun You and Eun Joo Kim have equally contributed to the study as corresponding authors.

${ }^{*}$ Corresponding authors: Joshua (Sung) H. You, 1 Yonseidae Kil, Wonju, Kangwon-do, 220-710, Korea. Tel.: +82 33760 2476; Fax: +82 337602496; E-mail: neurorehab@yonsei.ac.kr; Eun Joo Kim, 58 Samgaksan-ro, Gangbuk-gu, Seoul, 142-070, Korea. Tel.: +82 2901 1633; Fax: +82 2901 1591; E-mail: silverzookim@gmail.com.
}

0928-7329/17/\$35.00 (C) 2017 - IOS Press and the authors. All rights reserved

This article is published online with Open Access and distributed under the terms of the Creative Commons Attribution NonCommercial License (CC-BY-NC 4.0). 


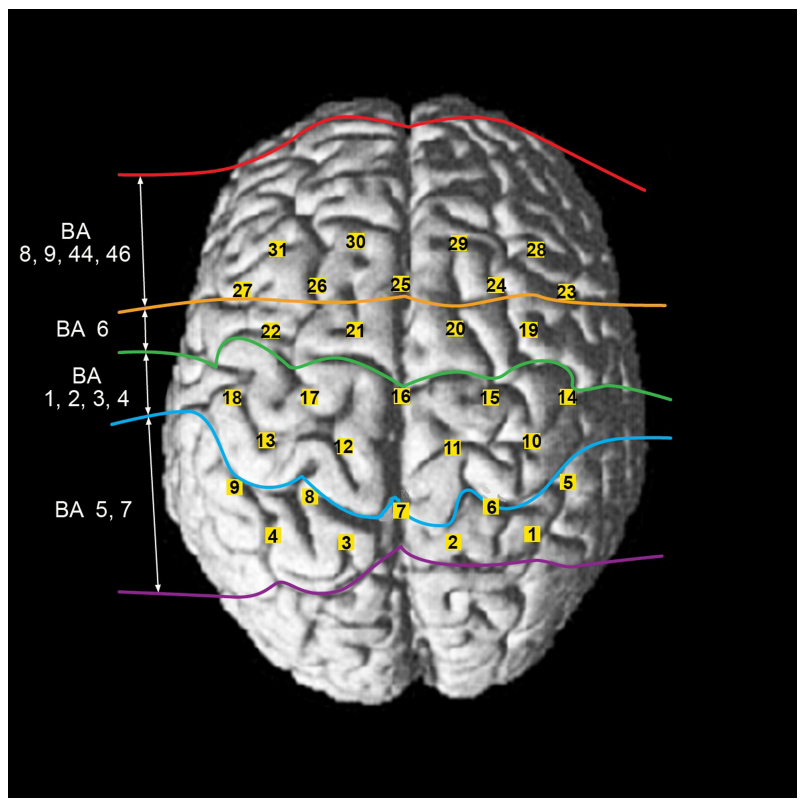

Fig. 1. Locations of the 31 channels and their corresponding cortical regions.

network is paramount for the improvement of rehabilitative efforts. Recently, Lee et al. [1] examined the effects of progressive walking-to-running therapy on gait kinematics in a child with decreased gait speed secondary to cerebral palsy. This study reported that walking-to-running therapy enhanced locomotor function and gait speed; however, cortical patterns in the locomotor network underlying improvements in gait speed remain unknown. It has been theorized that locomotion is produced by the cooperative activity of spinal pattern generator neurons, motor centers of the brain stem, and motor neurons located in the medial primary motor cortex [2-4]. Functional near-infrared spectroscopic (fNIRS) imaging is an advanced neuroimaging technique that has been utilized to identify cortical activation patterns and locomotor networks involved in continuous walking. In one study, fNIRS was used to identify bilateral increases in oxygenated hemoglobin $(\mathrm{OxyHb})$ in the medial sensorimotor cortex and supplementary motor area during continuous walking on a treadmill at a speed of $1 \mathrm{~km} / \mathrm{h}$ [5]. Suzuki et al. used fNIRS to evaluate cortical adaptation of the locomotor network in response to increases in gait speed over a range of 3-9 km/h and identified the involvement of the premotor cortex (PMC) and prefrontal cortex (PFC) [6]. Nevertheless, information about the cortical activation patterns and locomotor networks that mediate different walking speeds in different populations is highly limited. Thus, the purpose of the present study was to examine adaptive locomotor networks and cortical activation associated with different walking speeds over a range of $1.5-3.0 \mathrm{~km} / \mathrm{h}$, which are speeds relevant to those observed in patients recovering from hemiparetic stroke.

\section{Materials and methods}

\subsection{Materials and procedure}

A healthy right-handed subject (female, 30 years of age) with no neuromusculoskeletal impairments was recruited to participate in this study. The subject provided written informed consent prior to participation. For fNIRS (LABNIRS, Shimadzu Co., Kyoto, Japan) recording, a 31-channel system with 20 
optodes (consisting of 10 emitter and 10 detectors) and an inter-optode distance of $30 \mathrm{~mm}$ was applied to the subject according to the international 10-20 system.

Initially, the subject was requested to stand still on the treadmill for $60 \mathrm{~s}$. The subject was then verbally instructed to "start" and was made to walk on the treadmill at speeds of $1.5,2.0,2.5$, and $3.0 \mathrm{~km} / \mathrm{h}$. The order of the four treadmill speeds was randomized. Protocols were implemented as block-schemed experiments, each including a 30-s task period and a 30-s rest period with a 15 -s rest before and after each task period repeated 5 times.

The fNIRS system was equipped with wavelengths of 780,805 , and $830 \mathrm{~nm}$ and recorded cortical activation related to different walking speeds. According to the international 10-20 system, $\mathrm{Cz}$ (cranial vertex) was first defined underneath the 16th channel between the 7th emitter and the 4th detector, and then the remaining optodes were applied to the regions of interest (ROIs, $12 \times 9 \mathrm{~cm}$ in size) including the PFC, supplementary motor area (SMA), PMC, primary sensorimotor cortex (SMC), and somatosensory association cortex (SAC). The locations of optodes were correctly configured using a 3D position-measuring system (FASTRAK, Polhemus, VT, USA). The acquired optical imaging data were normalized to the standard stereotaxic space of the Montreal Neurological Institute (MNI) brain template, using NIRS-statistical parametric mapping software (NIRS-SPM, http://bisp.kaist.ac.kr/NIRSSPM). Anatomical ROIs were clinically correlated with Brodmann areas (BAs), which corresponded to BAs 1, 2, 3, and 4 (SMC); BA 6 (PMC); BAs 8, 9, 44, and 46 (PFC); and BAs 5 and 7 (SAC, Fig. 1).

\subsection{Data analysis}

The NIRS-SPM software package was utilized to analyze fNIRS data in a MATLAB environment (The Mathworks, Inc., MA, USA). Any artificial noise was corrected using Gaussian smoothing with a full width at a half maximum of $2 \mathrm{~s}$ [7]. Any signal distortions associated with movement artifacts were corrected by the wavelet minimum description length based on a detrending algorithm. A general linear model (GLM) analysis was executed with a canonical hemodynamic response curve to model the hypothesized $\mathrm{OxyHb}$ response recorded during different walking speeds [7]. SPM t-statistic maps were computed at an uncorrected threshold of $p<0.00001$.

To quantify cortical activation, fNIRS data recorded from the latter $20 \mathrm{~s}$ of the 30 -s task periods and from the middle $20 \mathrm{~s}$ of 30-s rest periods were used for our analyses because of a documented 3-5 s delay in the response of hemoglobin oxygenation to tasks [5]. Changes in hemoglobin oxygenation $(\triangle \mathrm{OxyHb})$ were then computed by subtracting $\mathrm{OxyHb}$ during the task period from OxyHb during the rest period for each channel. Additionally, regional cortical activation ratios in each channel were defined by computing $\Delta \mathrm{OxyHb}$ in each channel/total $\Delta \mathrm{OxyHb}$ from all 31 channels $\times 100$ to determine the relative adaptive contribution of localized cortical activation in response to changes in walking.

\section{Results}

\subsection{Comparison of fNIRS data mapping during different walking speeds}

Figure 2 illustrates cortical activation patterns during the different walking speeds in the test subject. At walking speeds of 1.5 and $2.0 \mathrm{~km} / \mathrm{h}$, activation was chiefly noted in the SMA and PMC. However, at a speed of $2.5 \mathrm{~km} / \mathrm{h}$, the SMC, PMC, SMA, and PFC were activated in a manner more indicative of globalized locomotor network activation. At a speed of $3.0 \mathrm{~km} / \mathrm{h}$, activation was primarily centered around the sensorimotor cortices and included the bilateral SMC, PMC, SMA, and PFC. 
Table 1

Regional OxyHb values in the bilateral hemispheres during different walking speeds

\begin{tabular}{|c|c|c|c|c|c|c|c|c|}
\hline \multirow{2}{*}{$\begin{array}{l}\text { Walking } \\
\text { speed }\end{array}$} & \multicolumn{2}{|c|}{$1.5 \mathrm{~km} / \mathrm{h}$} & \multicolumn{2}{|c|}{$2.0 \mathrm{~km} / \mathrm{h}$} & \multicolumn{2}{|c|}{$2.5 \mathrm{~km} / \mathrm{h}$} & \multicolumn{2}{|c|}{$3.0 \mathrm{~km} / \mathrm{h}$} \\
\hline & Left & Right & Left & Right & Left & Right & Left & Right \\
\hline PFC & 0.003268 & 0.003008 & 0.002234 & 0.002690 & 0.004328 & 0.003857 & 0.004610 & 0.002291 \\
\hline SMA & 0.007155 & 0.007063 & 0.011070 & 0.009579 & 0.012210 & 0.012958 & 0.011030 & 0.009825 \\
\hline PMC & 0.004052 & 0.004523 & 0.004249 & 0.002623 & 0.006875 & 0.006732 & 0.004979 & 0.003175 \\
\hline SMC & 0.005498 & 0.008492 & 0.006846 & 0.005384 & 0.007739 & 0.006912 & 0.008329 & 0.008925 \\
\hline SAC & 0.003221 & 0.001971 & 0.002047 & 0.004398 & 0.006927 & 0.009147 & 0.006434 & 0.002950 \\
\hline
\end{tabular}

Table 2

Regional cortical activation ratios in the bilateral hemispheres during different walking speeds

\begin{tabular}{|c|c|c|c|c|c|c|c|c|}
\hline \multirow{2}{*}{$\begin{array}{l}\text { Walking } \\
\text { speed }\end{array}$} & \multicolumn{2}{|c|}{$1.5 \mathrm{~km} / \mathrm{h}$} & \multicolumn{2}{|c|}{$2.0 \mathrm{~km} / \mathrm{h}$} & \multicolumn{2}{|c|}{$2.5 \mathrm{~km} / \mathrm{h}$} & \multicolumn{2}{|c|}{$3.0 \mathrm{~km} / \mathrm{h}$} \\
\hline & Left & Right & Left & Right & Left & Right & Left & Right \\
\hline PFC & 4.60 & 4.23 & 3.45 & 4.15 & 2.49 & 2.22 & 4.07 & 2.02 \\
\hline SMA & 10.07 & 9.94 & $17.09^{*}$ & 14.79 & 7.02 & $\mathbf{7 . 4 5}^{*}$ & $9.73^{*}$ & 8.67 \\
\hline PMC & 5.70 & 6.37 & 6.56 & 4.05 & 3.95 & 3.87 & 4.39 & 2.80 \\
\hline SMC & 7.74 & $11.95^{*}$ & 10.57 & 8.31 & 4.45 & 3.97 & 7.87 & 7.35 \\
\hline SAC & 4.53 & 2.77 & 3.16 & 6.79 & 3.98 & 5.26 & 5.68 & 2.60 \\
\hline
\end{tabular}

* represents the most activated cortical area among the predetermined ROIs within each walking speed.

\begin{tabular}{|l|l|l|l|}
\hline Walking speed $1.5 \mathrm{~km} / \mathrm{h}$ & Walking speed $2.0 \mathrm{~km} / \mathrm{h}$ & Walking speed $2.5 \mathrm{~km} / \mathrm{h}$ & Walking speed $3.0 \mathrm{~km} / \mathrm{h}$ \\
\hline $\mathrm{A}$ & \\
$\mathrm{P}$ &
\end{tabular}

Fig. 2. Patterns of cortical activation during different walking speeds.

\subsection{Comparison of regional OxyHb values during different walking speeds}

As walking speed increased, OxyHb intensity was more highly concentrated in the PFC and SMC. Specifically, OxyHb in the PFC and SMC peaked in the $3 \mathrm{~km} / \mathrm{h}$ condition relative to other speeds. At $2.5 \mathrm{~km} / \mathrm{h}$, OxyHb was highest in the SMA and activation in the PMC and SAC peaked relative to other speeds.

In the $1.5 \mathrm{~km} / \mathrm{h}$ condition, the SMC exhibited the highest cortical activation ratio. For the remaining conditions $(2.0,2.5$, and $3.0 \mathrm{~km} / \mathrm{h})$, the highest cortical activation ratio was observed in the SMA.

\section{Discussion}

The present fNIRS study is the first to present preliminary evidence for alternative patterns of cortical activation in response to different walking speeds. As walking speed increased, OxyHb was concentrated in the PFC and SMC and more globalized locomotor network activation was observed. The present neuroimaging data are consistent with previous studies $[6,8,9]$ that demonstrated important functional roles 
of PFC [6], nucleus accumbens, and ventral tegmental area [8] in locomotion. Specifically, increased OxyHb levels were observed in the PFC during running at a speed of $9 \mathrm{~km} / \mathrm{h}$ [6]. Tzschentke and Schmidt [8] reported that the medial PFC, nucleus accumbens, and ventral tegmental area were activated during locomotor behavior. Similarly, the PFC activation was improved in the cats with unilateral sensorimotor or frontal cortex ablation, which was functionally correlated with locomotor recovery [9]. From a theoretical perspective, increased walking speed may have increased cognitive-locomotor demand and required the adaptation of executive locomotor control at various levels of movement processing, selection, maintenance, updating, and rerouting. It has been theorized that the PFC mediates cognitive attention and the adaptive processing required for maintaining posture within the base of support and executing appropriate leg movements when walking speed increases. Indeed, increased PFC activity has been associated with intentional locomotor control [10-13], such that enhanced PFC activation as evidenced in the present $\mathrm{OxyHb}$ data reflects neurophysiological response to increased cognitive-locomotor demand. Future studies are warranted to validate our observations and further elucidate the neurophysiological mechanism underlying cognitive-locomotor control.

Present fNIRs data showed that the SMA was activated during increased walking speeds. The SMA is believed to contribute to locomotor control [5,14], postural control [15], and phasic postural adjustments [16]. In tandem with the PFC, the SMA is an important region in human locomotion and finetuning balance with increased walking speed. In previous literature, spinal locomotor pattern generators have been primarily thought to mediate rhythmic locomotor movement [17-19]. However, based on this assumption, we should have observed a decrease in cortical activation associated with increased walking speeds. In the present study, fNIRS data indicated an increase in cortical activation proportional to the increase in walking speed. Supraspinal modulation may have been recruited to respond to a new contextual locomotor environment (e.g., randomly increasing speed), and thus required more globalized pattern of cortical motor network activation.

\section{Conclusion}

The present study demonstrates that globalized locomotor network activation occurs during faster walking speeds in a healthy subject. As walking speed increased, OxyHb was more concentrated in the PFC and SMC. A better understanding of the mechanisms underlying locomotor network recruitment and activation will provide important insights for preclinical and clinical research alike and will ultimately lead to an improvement in the neurorehabilitation of neurological conditions such as stroke and cerebral palsy. Additional studies are required to capitalize on the full utility of fNIRS technology, to validate the findings presented herein, and to elucidate the neuroplastic changes associated with locomotor recovery in neurological populations.

\section{Acknowledgments}

This study was supported by the Department of Translational Research at the Center for Rehabilitation Robots, Korea National Rehabilitation Center (Grant no. NRCTR-IN14001).

\section{Conflict of interest}

None to report. 


\section{References}

[1] Lee NG, Jeong SJ, You JS, Cho KH, Lee TH. Effects of the progressive walking-to-running technique on gait kinematics, ultrasound imaging, and motor function in spastic diplegic cerebral palsy - an experimenter-blind case study. NeuroRehabilitation. 2013; 32(1): 17-26.

[2] Armstrong DM. The supraspinal control of mammalian locomotion. J Physiol. 1988; 405: 1-37.

[3] Drew T. Motor cortical cell discharge during voluntary gait modification. Brain Res. 1988; 457(1): 181-7.

[4] Mori S, Matsuyama K, Mori F, Nakajima K. Supraspinal sites that induce locomotion in the vertebrate central nervous system. Adv Neurol. 2001; 87: 25-40.

[5] Miyai I, Tanabe HC, Sase I, Eda H, Oda I, Konishi I, et al. Cortical mapping of gait in humans: a near-infrared spectroscopic topography study. Neuroimage. 2001; 14(5): 1186-92.

[6] Suzuki M, Miyai I, Ono T, Oda I, Konishi I, Kochiyama T, et al. Prefrontal and premotor cortices are involved in adapting walking and running speed on the treadmill: an optical imaging study. Neuroimage. 2004; 23(3): 1020-6.

[7] Ye JC, Tak S, Jang KE, Jung J, Jang J. NIRS-SPM: statistical parametric mapping for near-infrared spectroscopy. Neuroimage. 2009; 44(2): 428-47.

[8] Tzschentke TM, Schmidt WJ. Functional relationship among medial prefrontal cortex, nucleus accumbens, and ventral tegmental area in locomotion and reward. Crit Rev Neurobiol. 2000; 14(2): 131-42.

[9] Hovda DA, Fenney DM. Amphetamine with experience promotes recovery of locomotor function after unilateral frontal cortex injury in the cat. Brain Res. 1984; 298(2): 358-61.

[10] Malouin F, Richards CL, Jackson PL, Dumas F, Doyon J. Brain activations during motor imagery of locomotor-related tasks: a PET study. Hum Brain Mapp. 2003; 19(1): 47-62.

[11] Pochon JB, Levy R, Poline JB, Crozier S, Lehericy S, Pillon B, et al. The role of dorsolateral prefrontal cortex in the preparation of forthcoming actions: an fMRI study. Cereb Cortex. 2001; 11(3): 260-6.

[12] Lau HC, Rogers RD, Ramnani N, Passingham RE. Willed action and attention to the selection of action. Neuroimage. 2004; 21(4): 1407-15.

[13] Lau HC, Rogers RD, Haggard P, Passingham RE. Attention to intention. Science. 2004; 303(5661): 1208-10.

[14] Fukuyama H, Ouchi Y, Matsuzaki S, Nagahama Y, Yamauchi H, Ogawa M, et al. Brain functional activity during gait in normal subjects: a SPECT study. Neurosci Lett. 1997; 228(3): 183-6.

[15] Massion J. Movement, posture and equilibrium: interaction and coordination. Prog Neurobiol. 1992; 38(1): 35-56.

[16] Viallet F, Massion J, Massarino R, Khalil R. Coordination between posture and movement in a bimanual load lifting task: putative role of a medial frontal region including the supplementary motor area. Exp Brain Res. 1992; 88(3): 674-84.

[17] Duysens J, Van de Crommert HW. Neural control of locomotion; The central pattern generator from cats to humans. Gait Posture. 1998; 7(2): 131-41.

[18] Miyai I, Yagura H, Oda I, Konishi I, Eda H, Suzuki T, et al. Premotor cortex is involved in restoration of gait in stroke. Ann Neurol. 2002; 52(2): 188-94.

[19] Wickelgren I. Teaching the spinal cord to walk. Science. 1998; 279(5349): 319-21. 